\title{
Spherically symmetric model stellar atmospheres and limb darkening
}

\section{Limb-darkening laws, gravity-darkening coefficients and angular diameter corrections for FGK dwarf stars ${ }^{\star}$}

\author{
H. R. Neilson ${ }^{1}$ and J. B. Lester ${ }^{2,3}$ \\ 1 Department of Physics \& Astronomy, East Tennessee State University, Box 70652, Johnson City, TN 37614, USA \\ e-mail: neilsonh@etsu.edu \\ 2 Department of Chemical and Physical Sciences, University of Toronto Mississauga, 3359 Mississauga, Ontario, L5L 156, Canada \\ 3 Department of Astronomy \& Astrophysics, University of Toronto, Toronto, Ontario, MSS 3H4, Canada \\ e-mail: lester@astro.utoronto.ca
}

Received 13 May 2013 / Accepted 24 June 2013

\begin{abstract}
Limb darkening is a fundamental ingredient for interpreting observations of planetary transits, eclipsing binaries, optical/infrared interferometry and microlensing events. However, this modeling traditionally represents limb darkening by a simple law having one or two coefficients that have been derived from plane-parallel model stellar atmospheres, which has been done by many researchers. More recently, researchers have gone beyond plane-parallel models and considered other geometries. We previously studied the limbdarkening coefficients from spherically symmetric and plane-parallel model stellar atmospheres for cool giant and supergiant stars, and in this investigation we apply the same techniques to FGK dwarf stars. We present limb-darkening coefficients, gravity-darkening coefficients and interferometric angular diameter corrections from Atlas and SAtlas model stellar atmospheres. We find that sphericity is important even for dwarf model atmospheres, leading to significant differences in the predicted coefficients.
\end{abstract}

Key words. stars: atmospheres - binaries: eclipsing - stars: evolution - planetary systems - techniques: interferometric

\section{Introduction}

One of the great astronomical advances of the past two decades has been the discovery and study of extrasolar planets via the transit method, i.e. from the minute drop of a star's flux as a planet passes in front of it. The transit not only constrains the planet's properties but also the star's properties, such as limb darkening. However, interpreting planetary transits typically assumes that limb darkening can be parametrized by a simple relation (Mandel \& Agol 2002) with a few free parameters that can be fit directly from the observations or assumed from model stellar atmospheres.

Limb darkening is important not only for understanding planetary transits (e.g. Croll et al. 2011), but also for interpreting optical interferometric observations (e.g. Davis et al. 2000) and microlensing observations (e.g. An et al. 2002) and eclipsing binary light curves (e.g. Bass et al. 2012). Like transit measurements, interferometric and microlensing observations are typically fit by simple limb-darkening laws with coefficients derived from model stellar atmospheres (Al-Naimiy 1978; Wade \& Rucinski 1985; van Hamme 1993; Claret 2000; Claret \& Bloemen 2011; Claret et al. 2012). However, these simple limbdarkening laws have become less suitable as the observations have improved. For example, Fields et al. (2003) showed that flux-normalized limb-darkening laws fit to Atlas plane-parallel

* Tables 2-17 and model intensity profiles are only available at the CDS via anonymous ftp to cdsarc.u-strasbg.fr (130.79.128.5) or via

http: //cdsarc.u-strasbg.fr/viz-bin/qcat?]/A+A/556/A86 model atmospheres disagreed with microlensing observations. Limb-darkening coefficients derived from planetary transit observations with large impact parameters differ more from the limb-darkening coefficients from model atmospheres, but the discrepancy still exists when the impact parameter is taken into account (Barros et al. 2012).

This discrepancy might be due to a number of physical processes, including granulation, multidimensional convection and/or the presence of magnetic fields in the stellar atmosphere. However, the simplest step is to assume a more realistic geometry for the model stellar atmospheres. Limb-darkening coefficients presented in the literature are based on two forms: plane-parallel model stellar atmospheres computed using the Atlas (Kurucz 1979) and Phoenix code (Hauschildt et al. 1999) and spherically symmetric model stellar atmospheres also computed from the Phoenix code (Sing 2010; Howarth 2011a; Claret \& Bloemen 2011; Claret et al. 2012). In particular, Claret \& Hauschildt (2003) and Claret et al. (2012, 2013) explored limb darkening using spherically symmetric Phoenix model stellar atmospheres specifically for main sequence stars. They also introduced the concept of "quasi-spherical" models, defined as the spherically-symmetric intensity profile restricted to inner part of the stellar disk $(\mu \geq 0.1)$, to compare limb-darkening coefficients with those from plane-parallel models.

In our previous study (Neilson \& Lester 2013, hereafter Paper I), we presented coefficients for six typical limb-darkening laws fit to the surface intensities for grids of plane-parallel and spherical model atmospheres (Lester \& Neilson 2008) representing red giant and supergiant stars. The intensities were for the 
wavebands of the Johnson-Cousins (Johnson \& Morgan 1953; Bessell 2005), CoRot (Auvergne et al. 2009) and Kepler (Koch et al. 2004) filters. We also computed gravity-darkening coefficients and interferometric angular diameter corrections. We found that the predicted limb-darkening coefficients computed from spherical model atmospheres differ from those computed from plane-parallel model atmospheres, which was not unexpected; the height of the atmospheres of red giants and supergiants relative to the stellar radius is many times greater than the relative heights of the atmospheres of main-sequence stars, i.e. the assumed model geometry is important. We found similar differences for the angular diameter corrections as a function of geometry but little difference between gravity-darkening coefficients as a function of geometry. While model atmosphere geometry is clearly important for understanding the extended atmospheres of red giant and supergiant stars, it is not as obvious that geometry also changes predictions for model stellar atmospheres of main sequence dwarf stars (e.g. Claret \& Hauschildt 2003).

In this work, we explore the role of model atmosphere geometry in understanding limb darkening in dwarf stars and compute limb-darkening coefficients, gravity-darkening coefficients and interferometric angular diameter corrections from grids of model stellar atmospheres of dwarf stars. In Sect. 2, we briefly describe the grids of model atmospheres used. In Sect. 3, we describe various limb-darkening laws and compare predicted limb-darkening coefficients, while in Sect. 4 we compute gravity-darkening coefficients. We present interferometric angular diameter corrections as a function of geometry in Sect. 5 and summarize our results in Sect. 6.

\section{Model stellar atmospheres}

The Atlas/SAtlas code was used to compute model stellar atmospheres assuming either plane-parallel or spherically symmetric geometry. Details of the code can be found in Lester \& Neilson (2008), Neilson \& Lester $(2011,2012)$ and Paper I. We computed model stellar atmospheres with parameters $3500 \mathrm{~K} \leq$ $T_{\text {eff }} \leq 8000 \mathrm{~K}$ in steps of $100 \mathrm{~K}$, and $4.0 \leq \log g \leq 4.75$ in steps of 0.25 . For the spherically symmetric models, which require an additional parameter, such as stellar mass, to characterize the atmosphere, we chose $M=0.2$ to $1.4 M_{\odot}$ in steps of $0.3 M_{\odot}$. For each model stellar atmosphere we compute intensities at each wavelength for 1000 uniformly spaced values of $\mu$, the cosine of the angle formed by the line-of-sight point on the stellar disk and the disk center, spanning $0 \leq \mu \leq 1$. Typically, Atlas models compute intensities at only seventeen angles (Kurucz 1979), but some have employed $100 \mu$-points (Claret \& Bloemen 2011). We compute intensity profiles for each model atmosphere for the BVRIH and $K$-bands as well as the CoRot and Kepler-bands. As an example, Fig. 1 shows the Kepler-band intensity profiles for plane-parallel and spherical models with $T_{\text {eff }}=5800 \mathrm{~K}, \log g=4.5$ and $M=1.1 M_{\odot}$. Using the wavebands outlined above, we compute limb-darkening coefficients, gravity-darkening coefficients and interferometric angular diameter corrections.

\section{Limb-darkening laws}

We consider the same six limb-darkening laws as in Paper I:

$\frac{I(\mu)}{I(\mu=1)}=1-u(1-\mu)$ Linear, (1)

$\frac{I(\mu)}{I(\mu=1)}=1-a(1-\mu)-b(1-\mu)^{2}$

Quadratic, (2)

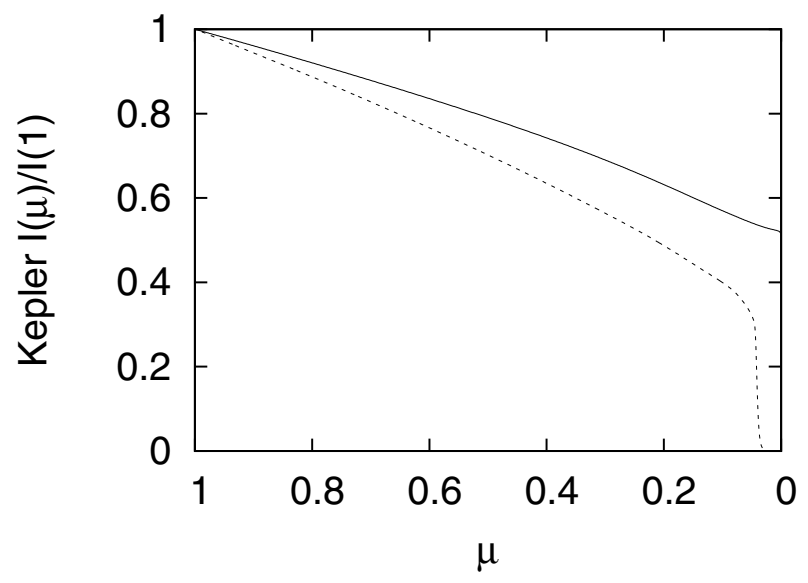

Fig. 1. Predicted Kepler-band intensity profiles for plane-parallel (solid line) and spherically symmetric (dotted line) model stellar atmosphere with $T_{\text {eff }}=5800 \mathrm{~K}, \log g=4.5$ and $M=1.1 M_{\odot}$.

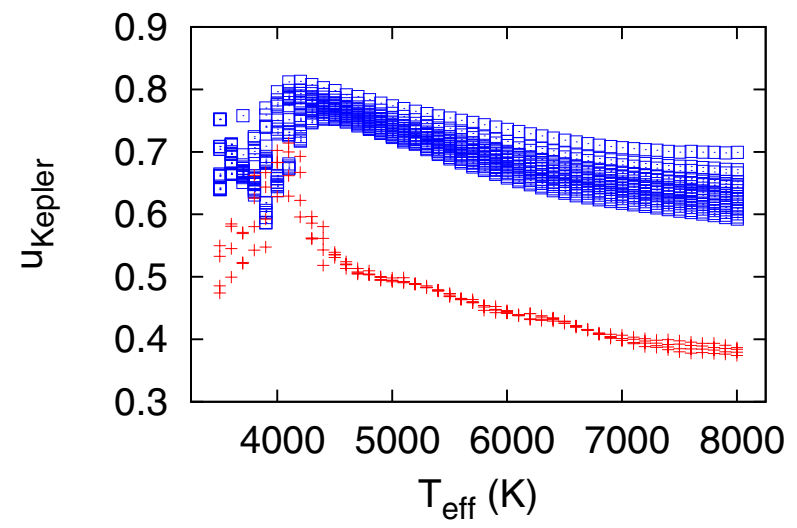

Fig. 2. The limb-darkening coefficient $u$, used in the linear law (Eq. (1)), applied to the Kepler photometric band. Crosses are the plane-parallel model stellar atmospheres, and the squares are the spherical models.

$\frac{I(\mu)}{I(\mu=1)}=1-c(1-\mu)-d(1-\sqrt{\mu})$

Square-Root, (3)

$\frac{I(\mu)}{I(\mu=1)}=1-\sum_{j=1}^{4} f_{j}\left(1-\mu^{j / 2}\right)$

4-Parameter, (4)

$\frac{I(\mu)}{I(\mu=1)}=1-g(1-\mu)-h \frac{1}{1-e^{\mu}}$

Exponential, (5)

$\frac{I(\mu)}{I(\mu=1)}=1-m(1-\mu)-n \mu \ln \mu$

Logarithmic. (6)

As in Paper I, we use a general least-squares fitting algorithm to compute the limb-darkening coefficients for each law in the $B V R I H$ - and $K$-bands as well as for the CoRot and Keplerbands. Using the Kepler-band as an example, Fig. 2 shows the best-fit limb-darkening coefficient for the linear law (Eq. (1)), Fig. 3 shows the coefficients for the quadratic (Eq. (2)) and square-root (Eq. (3)) laws, Fig. 4 shows the coefficients for the exponential (Eq. (5)) and logarithmic (Eq. (6)) laws and Fig. 5 shows the coefficients for the Claret (2000) four-parameter law (Eq. (4)).

The results shown in Fig. 2 demonstrate how the geometry of the model atmosphere affects the best-fit linear Keplerband limb-darkening coefficients, with squares representing fits 
H. R. Neilson and J. B. Lester: Limb darkening in red giant stars
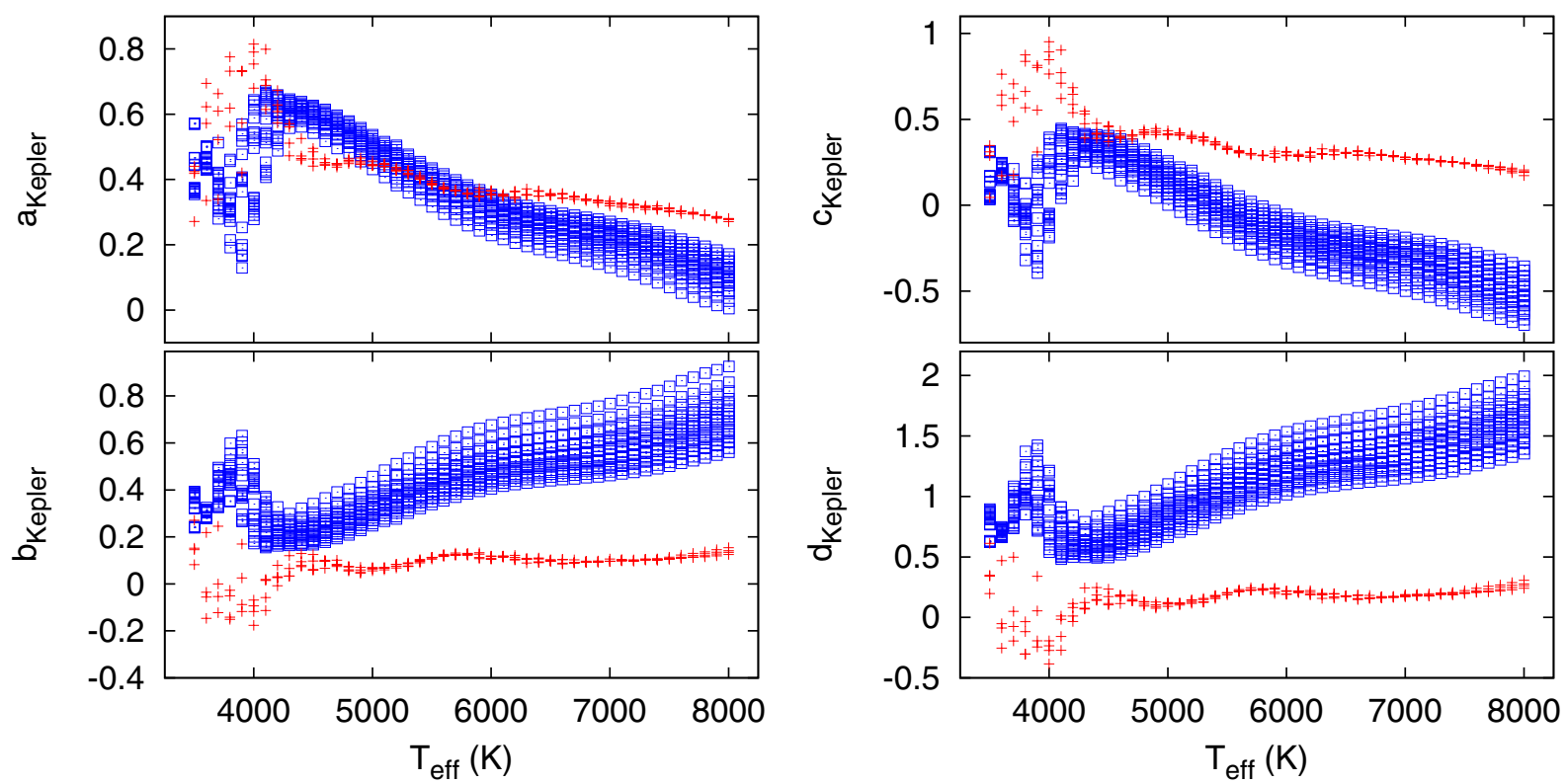

Fig. 3. Limb-darkening coefficients $a$ and $b$ used in the quadratic law (Eq. (2)) (left panel), and the coefficients $c$ and $d$ used in the square-root law (Eq. (3)) (right panel), all applied to the Kepler photometric band. The symbols have the same meanings as in Fig. 2.
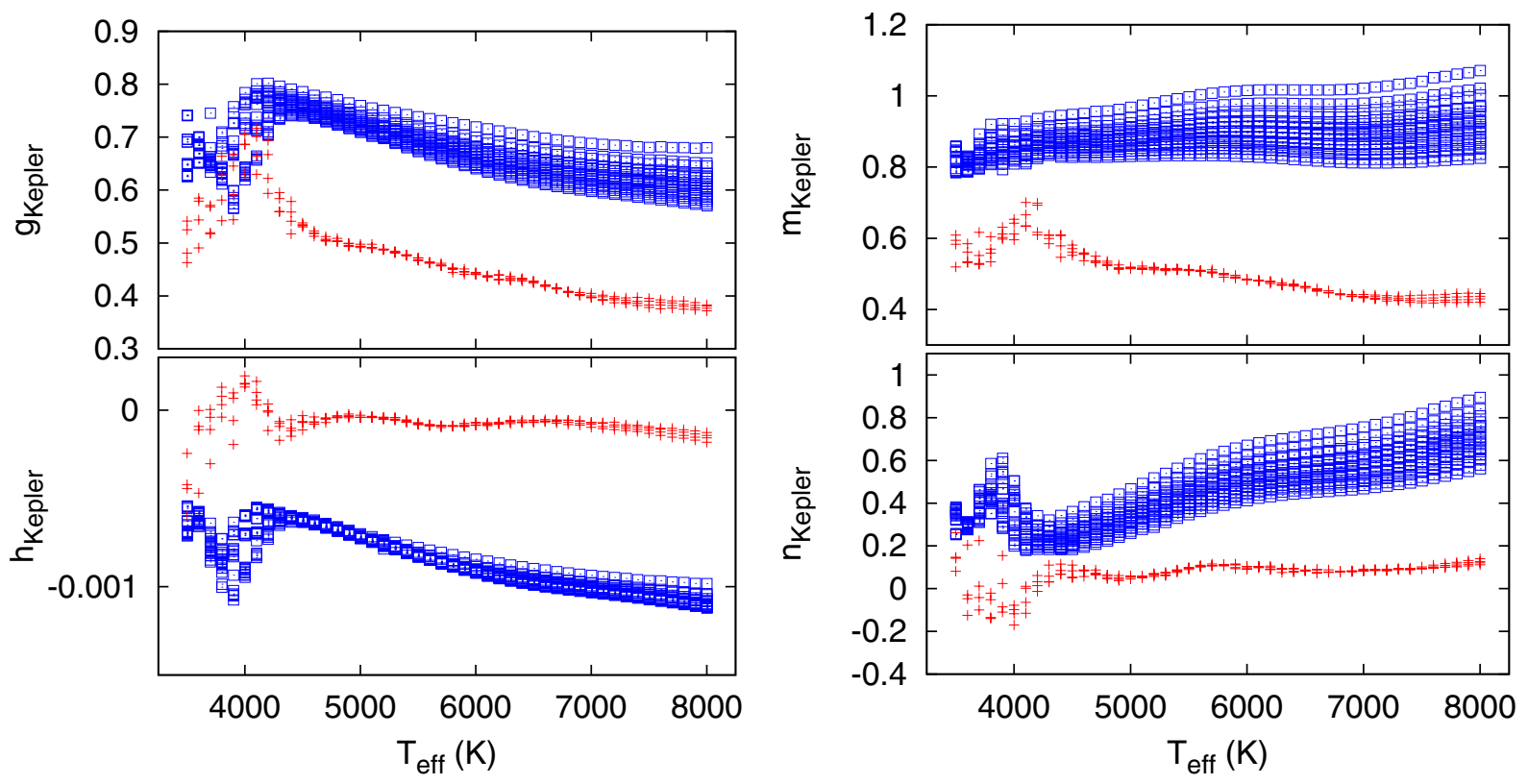

Fig. 4. Limb-darkening coefficients $g$ and $h$ used in the exponential law (Eq. (5)) (left panel), and the coefficients $m$ and $n$ used in the logarithmic law (Eq. (6)) (right panel), all applied to the Kepler photometric band. The symbols have the same meanings as in Fig. 2.

to the spherically symmetric model atmospheres and crosses representing fits to the plane-parallel models. The values of the $u$-coefficient for the spherical models are larger than those for the planar models, particularly for models with $T_{\text {eff }}>4500 \mathrm{~K}$. At these higher effective temperatures the difference due to geometry, $\Delta u_{\text {Kepler }}$, is $\sim 0.3$. There is also a greater spread for the spherical model coefficients at a given effective temperature. This is caused by the spherical models being defined by three parameters, with mass and radius being separated, as opposed to the two parameters for plane-parallel model atmospheres, where mass and radius are combined in the surface gravity. At $T_{\text {eff }}<4500 \mathrm{~K}$ the $u$-coefficients computed for both geometries shift to similar values. A likely cause of this change relative to the higher effective temperatures is the shift in dominant opacities from $\mathrm{H}^{-}$ to $\mathrm{TiO}$.
The more complex limb-darkening laws have similar differences between coefficients from plane-parallel and spherically symmetric models. For the quadratic and square-root laws, the coefficients of the linear term ( $a$ and $c$, respectively) shows similar behavior as a function of effective temperature as does the $u$-coefficients, while the coefficients of the non-linear terms ( $b$ and $d$ ) appear correlated to the coefficients of the linear terms, as was seen previously for other laws (Fields et al. 2003; Neilson \& Lester 2011, 2012).

For the exponential and logarithmic laws, the best-fit coefficients again differ as a function of model atmosphere geometry. The limb-darkening coefficients also appear to be correlated for each law. It is notable that the best-fit $m$-coefficients of the logarithmic law from spherically symmetric models are approximately constant with respect to effective temperature, whereas 

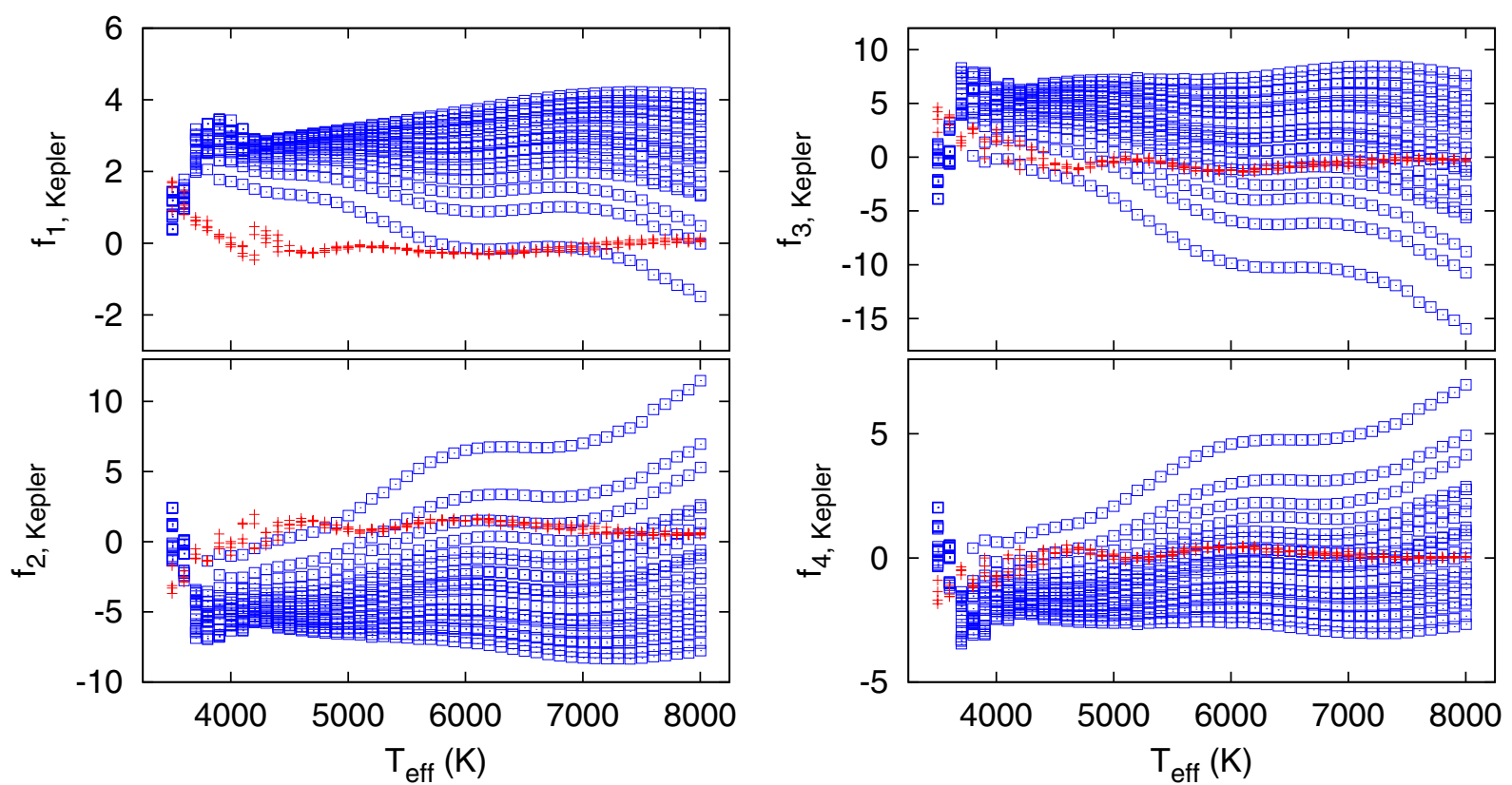

Fig. 5. Limb-darkening coefficients $f_{1}, f_{2}, f_{3}$ and $f_{4}$ used in the Claret (2000) four-parameter law, Eq. (4), applied to the Kepler photometric band. The symbols are the same as in Fig. 2.

the non-linear term is not constant. The limb-darkening coefficients from spherically symmetric models for both exponential and logarithmic laws vary significantly for any given effective temperature, suggesting the coefficients are sensitive to the mass and gravity of a model stellar atmosphere.

The best-fit coefficients for the Claret (2000) four-parameter limb-darkening laws do not agree for spherical and planeparallel models. For effective temperatures greater than $4000 \mathrm{~K}$, the limb-darkening coefficient $f_{1}$ varies from -2 to +4 for the spherical models but only from -0.5 to 0.5 for the plane-parallel models. The dramatic difference is due to the more complex structure of spherically symmetric model intensity profiles, even when considering the smaller atmospheric extensions for models used in this work as opposed to those considered in Paper I, which indicates that even this more sophisticated limb-darkening law is not ideal for fitting spherically symmetric model intensity profiles.

Figures 2-5 demonstrate that the best-fit coefficients from spherical models differ from those computed from planar models, but these figures do not quantify the fits for either geometry. To do this, we employ the parameter defined in Paper I,

$\Delta_{\lambda} \equiv \sqrt{\frac{\sum\left[I_{\text {model }}(\mu)-I_{\text {fit }}(\mu)\right]^{2}}{\sum\left[I_{\text {fit }}(\mu)\right]^{2}}}$,

to measure the difference for every model between the computed intensity distribution and the best fit to those intensities for each limb-darkening law. Unfortunately, as we showed in Paper I, the computed error depends on how the models are sampled and the number of intensity points. If one fits intensity profiles for $\mu$-points near the center of the stellar disk then the limb-darkening coefficients and predicted errors differ from limb-darkening coefficients and errors predicted from a sample of $\mu$-points near the edge of the stellar disk. However, we can predict the relative quality of fits as a function of geometry. We show in Fig. 6 the predicted errors for each limb-darkening law as a function of effective temperature.

As expected, Fig. 6 shows that all six limb-darkening laws fit the plane-parallel model atmosphere intensity profiles better than intensity profiles from spherical models. The definition of plane-parallel radiative transfer (Feautrier 1964) assumes that $I(\mu) \propto \mathrm{e}^{-\tau / \mu}$, where $\tau$ is the monochromatic optical depth. As $\mu \rightarrow 0$, then $I(\mu) \rightarrow 0$, i.e. the intensity and the derivative of the intensity, $\mathrm{d} I / \mathrm{d} \mu$, both change monotonically. These properties allow simple limb-darkening laws to fit plane-parallel model intensity profiles well.

For spherically symmetric model atmospheres the radiative transfer is calculated for a set of rays along the line-of-sight between the observer and points on the stellar disk. The rays nearer the center of the stellar disk come from depths that are assumed to be infinitely optically thick. The rays farther from the center of the stellar disk penetrate to depths where the optical depth is assumed never to reach infinity (Rybicki 1971; Lester \& Neilson 2008), although the rays can reach extremely large optical depths. Rays located toward the limb of the star can penetrate the tenuous outer atmosphere, never reaching large optical depths. As a result, the computed intensity profiles have a point of inflection (see Fig. 1) where the intensity derivative, $\mathrm{d} I / \mathrm{d} \mu$, is not changing monotonically, which prevents the simple limbdarkening laws from fitting as well.

While it is difficult to draw conclusions from the predicted errors, we can reliably state that the linear and exponential limbdarkening laws do not fit the spherical model atmospheres. The predicted errors for those limb-darkening laws range from 0.05 to 0.2 and are significantly greater than the errors for the fits to plane-parallel models. The best-fitting relations are the squareroot law and the four-parameter limb-darkening law of Claret (2000), which have errors less than 0.08 .

Another thing to note is that based on fits to plane-parallel model atmospheres, Diaz-Cordoves et al. (1995) suggested that the square-root law is more adequate for fitting hotter stars $\left(T_{\text {eff }}>8000 \mathrm{~K}\right)$, although they were unclear which law is preferred for cooler stars. For spherical model atmospheres we find that the predicted errors for the square-root limb-darkening law are less than the errors for the quadratic law, making the former the clear preference. Also, the quadratic limb-darkening law is of particular interest because it is the most commonly 
H. R. Neilson and J. B. Lester: Limb darkening in red giant stars
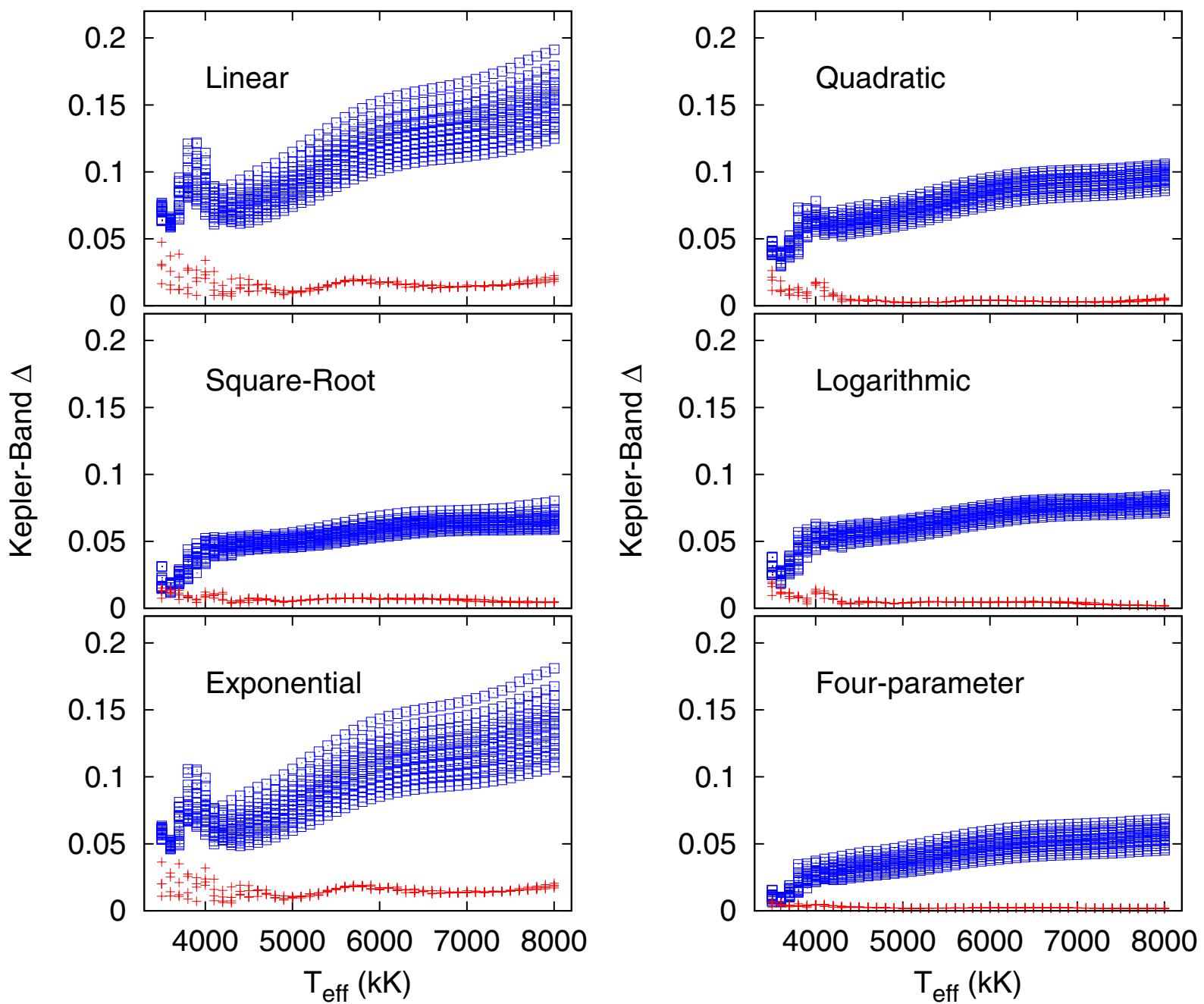

Fig. 6. The error of the best-fit limb-darkening relation, defined by Eq. (7), for every model atmosphere (crosses represent plane-parallel models, squares spherical models) for each of the six limb-darkening laws at Kepler-band wavelengths.

used limb-darkening law for analyzing planetary transit observations (Mandel \& Agol 2002). However, numerous comparisons of quadratic limb-darkening laws fit directly to observations and those fit to model stellar atmospheres suggest disagreement for a number of cases (Howarth 2011b). The results presented here suggest it may be advantageous to consider fitting transit observations with a square-root limb-darkening law or the more accurate four-parameter limb-darkening law.

\section{Gravity-darkening coefficients}

Claret \& Bloemen (2011) computed wavelength-dependent gravity-darkening coefficients from Atlas plane-parallel model stellar atmospheres based on the analytic relation developed by Bloemen et al. (2011). In Paper I we used this same prescription for both plane-parallel and spherically symmetric model stellar atmospheres to compute gravity-darkening coefficients for cool giant stars, and we found that model geometry played a negligible role in determining gravity-darkening coefficients except for $T_{\text {eff }}<4000 \mathrm{~K}$. At the cooler effective temperatures, the spherically symmetric model gravity-darkening coefficients are predicted to be vary significantly, and are up to an orderof-magnitude greater than those predicted from plane-parallel model atmospheres.
We repeat that analysis here for our higher gravity model stellar atmospheres. As described by Bloemen et al. (2011), the gravity-darkening coefficient, $y(\lambda)$ for a star is

$y(\lambda)=\left(\frac{\partial \ln I(\lambda)}{\partial \ln g}\right)_{T_{\mathrm{eff}}}+\left(\frac{\mathrm{d} \ln T_{\mathrm{eff}}}{\operatorname{dln} g}\right)\left(\frac{\partial \ln I(\lambda)}{\partial \ln T_{\mathrm{eff}}}\right)_{g}$.

As described in Paper I, von Zeipel (1924) showed that $T_{\text {eff }} \sim$ $\left(g_{\text {eff }}\right)^{\beta_{1} / 4}$, where $\beta_{1} \equiv \mathrm{d} \ln T_{\text {eff }} / \mathrm{d} \ln g$. As previously, we assume $\beta_{1}=0.2$ for models with $T_{\text {eff }}<7500 \mathrm{~K}$ and $\beta_{1}=1$ otherwise. Using these constant values for $\beta_{1}$ provides only a limited analysis of the gravity-darkening because $\beta_{1}$ is a function of effective temperature, but assuming these two values does enable us to gain some perspective on the role of model atmosphere geometry. The other terms are the partial derivatives of the wavelengthdependent intensity with respect to gravity and effective temperature, respectively.

We compute the two intensity derivatives and predicted gravity-darkening coefficients for our grids of plane-parallel and spherically symmetric model atmospheres and plot the predicted values in Fig. 7 for the Kepler waveband. The predicted derivatives and gravity-darkening coefficients are similar to those computed in Paper I, for which there is little difference between spherically symmetric and plane-parallel model predictions for effective temperatures greater than $4000 \mathrm{~K}$. The spherical and 

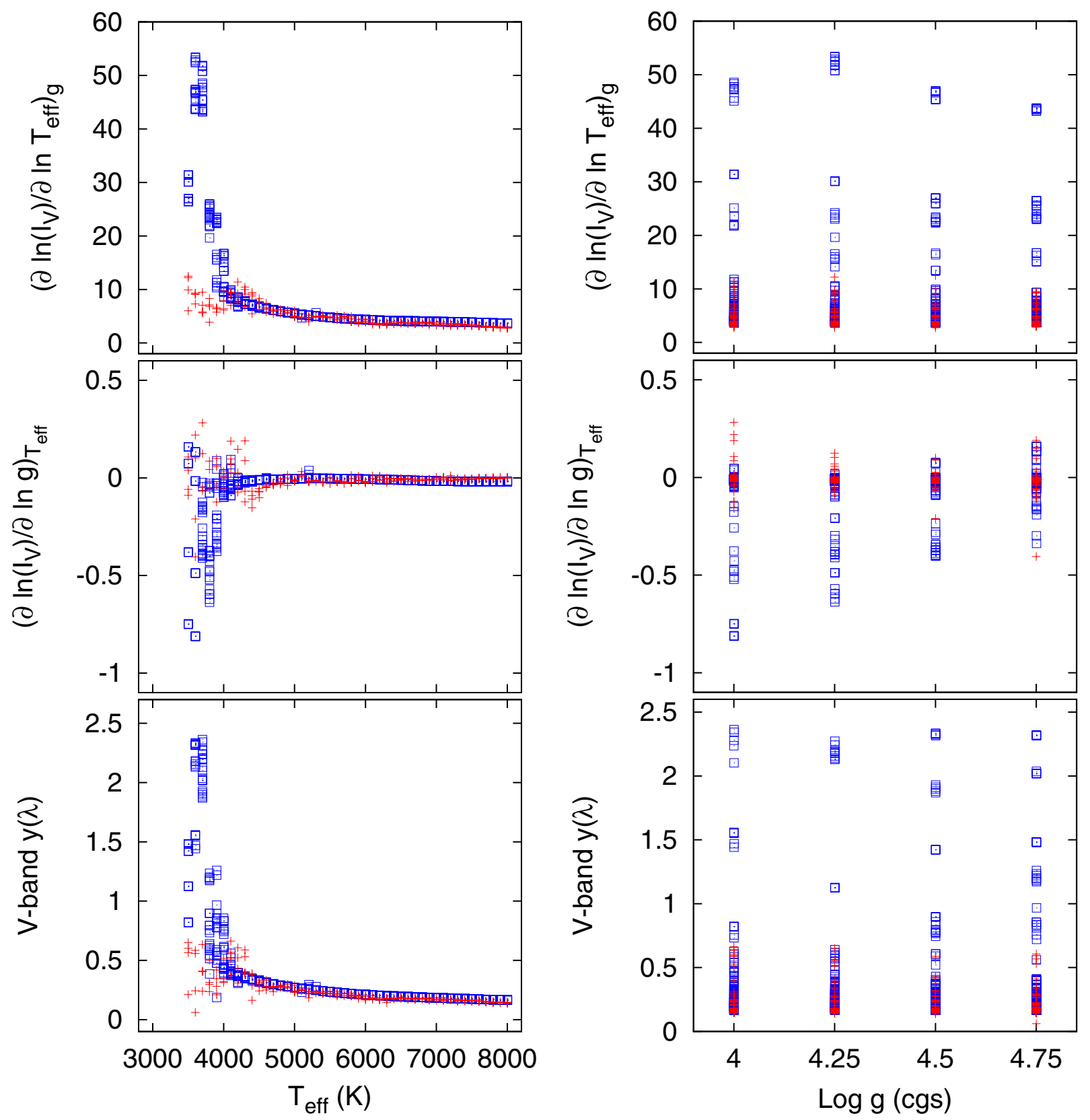

Fig. 7. $V$-band central intensity derivatives and gravity-darkening coefficients as function of effective temperature (left) and gravity (right) computed from plane-parallel (red crosses) and spherically symmetric (blue squares) model stellar atmospheres.

planar predictions then diverge for cooler effective temperatures. However, the range of values for the spherical model predictions is less for the higher gravity models explored in this work relative to the lower gravity models studied in Paper I.

\section{Interferometric angular diameter corrections}

Interferometry provides precise measurements of stellar angular diameters. However, stellar interferometry measures the combination of angular diameter and intensity profile and the two quantities are degenerate. One route to break the degeneracy is to assume a uniform intensity profile and measure the uniform-disk angular diameter. The limb-darkened angular diameter can then be predicted from the uniform-disk angular diameter using corrections computed from stellar atmosphere models (Davis et al. 2000).
Another technique for measuring limb-darkened angular diameters is to assume a simple limb-darkening law and coefficients from model stellar atmospheres to fit the interferometric observations (e.g. Boyajian et al. 2012). However, this technique might also predict incorrect angular diameters because plane-parallel model atmospheres are typically used for fitting limb-darkening coefficients. We can assess the potential error of assuming plane-parallel limb-darkening coefficients to fit the angular diameter by comparing predicted angular diameter corrections from spherically symmetric model stellar atmospheres with those from plane-parallel models.

In Fig. 8 we plot the $V$ - and $K$-band angular diameter corrections as a function of effective temperature and gravity for both spherical and planar model atmospheres. The $V$-band corrections vary from 0.93 to 0.97 for the plane-parallel model atmospheres and from 0.92 to 0.95 for spherical models. The 

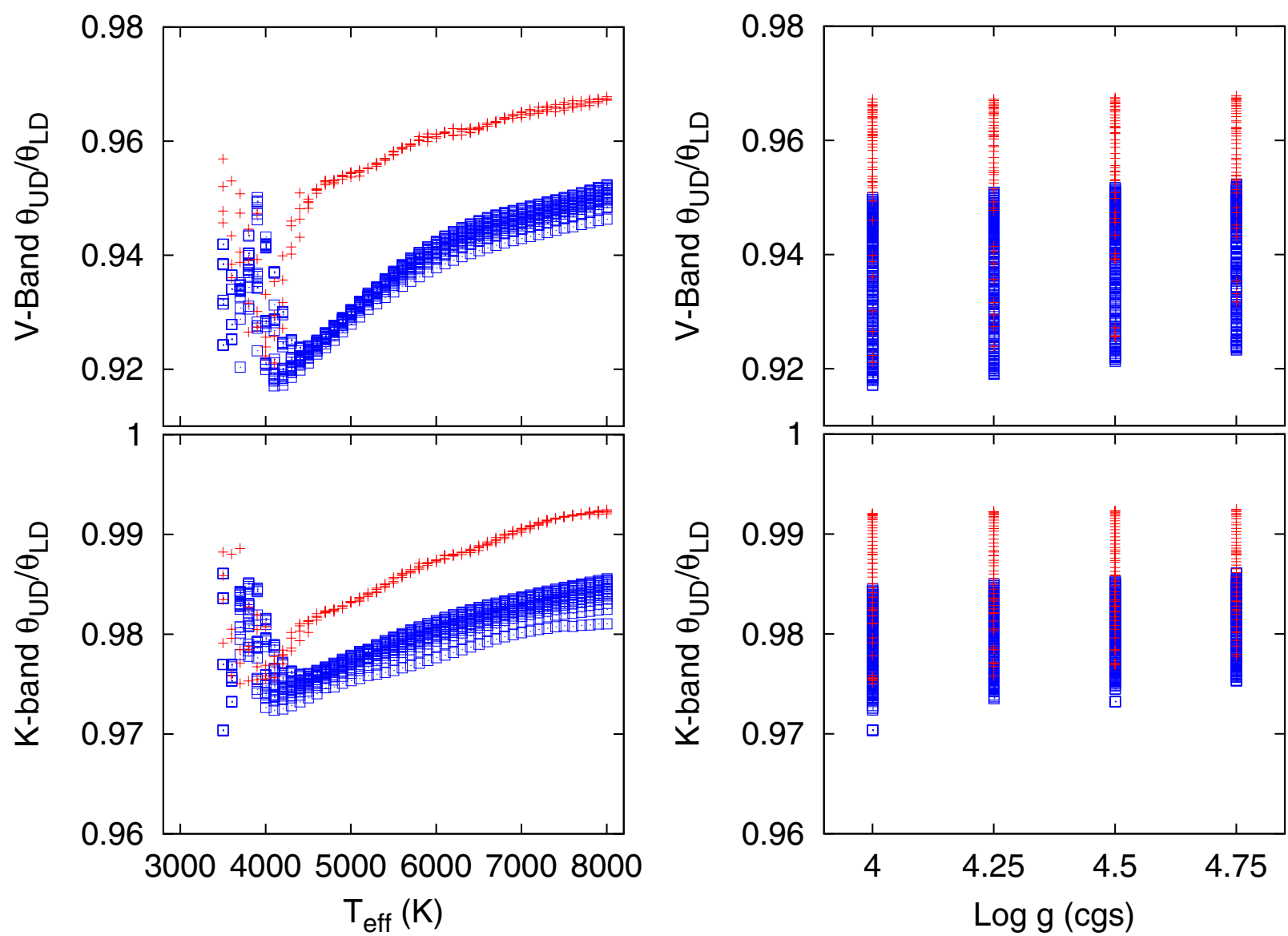

Fig. 8. Interferometric angular diameter correction computed in $V$-band (top) and $K$-band (bottom) as functions of effective temperature (left) and gravity $(r i g h t)$. Corrections computed from plane-parallel model atmospheres are denoted with red x's and spherically symmetric models blue squares.

difference is more apparent if one considers stellar atmospheres with $T_{\text {eff }}>4500 \mathrm{~K}$, where the difference between plane-parallel and spherical model corrections is about 0.01 to 0.02 . This suggests that employing plane-parallel model corrections for measuring stellar angular diameters from interferometric observations will lead to a 1 to $2 \%$ underestimate of the angular diameter.

Similarly, the $K$-band corrections also vary as a function of model atmosphere geometry; plane-parallel models suggest values of $\theta_{\mathrm{UD}} / \theta_{\mathrm{LD}}=0.98$ to 0.99 while spherical models suggest $\theta_{\mathrm{UD}} / \theta_{\mathrm{LD}}=0.97$ to 0.985 . Again, using plane-parallel model corrections to fit $K$-band interferometric observations will underestimate the actual angular diameter by about $1 \%$. Thus, for precision measurements of angular diameters, hence fundamental stellar parameters from optical interferometry, one should employ more physically representative spherical model atmospheres. This appears to be the case even for main sequence stars with large gravities and small atmospheric extensions.

\section{Summary}

In this work, we followed up on the study of Paper I to measure how model stellar atmosphere geometry affects predicted limb-darkening coefficients, gravity-darkening coefficients and interferometric angular diameter corrections for main sequence FGK dwarf stars. As in Paper I, we find significant differences between predictions from plane-parallel and spherically symmetric model atmospheres computed with the Atlas/SAtlas codes. The results in this article are surprising because geometry is believed to be not important for stars with smaller atmospheric extension, i.e. main sequence stars with $\log g \geq 4$. As atmospheric extension gets smaller, defined as the ratio of the atmospheric depth to stellar radius, then it is expected that a spherical model atmosphere should appear more and more like a planeparallel model atmosphere. However, even for small atmospheric extension models, we find differences in predicted intensity profiles, hence differences in limb-darkening and angular diameter corrections.

As in Paper I, there is negligible difference between gravitydarkening coefficients predicted from planar and spherical model atmospheres. This is because gravity-darkening coefficients depend heavily on the central intensity of the star, not the entire intensity profile. The central intensity is approximately a function of the effective temperature at $\tau_{\text {Ross }}=1$ according to the Eddington-Barbier relation (Mihalas 1978). Lester \& Neilson (2008) showed that the atmospheric temperature structure computed from plane-parallel and spherically symmetric model atmospheres for the same effective temperature and gravity primarily differs closer to the surface, $\tau_{\text {Ross }}<2 / 3$, and converges as $\tau \rightarrow \infty$. Because the computed temperatures at depth are very similar for the two geometries, the central intensity is also similar for both model geometries, making the gravity-darkening coefficients insensitive to model geometry. However, geometry is important for stars with $T_{\text {eff }}<4000 \mathrm{~K}$, which is due to differences in the opacity structure and convection, which lead to changes in the temperature structure.

Angular diameter corrections do vary as a function of geometry. The corrections account for the degeneracy between the 
Table 1. Summary of limb-darkening coefficient, gravity-darkening coefficient and interferometric angular diameter correction tables found online.

\begin{tabular}{lll}
\hline \hline Name & Geometry & Type \\
\hline Table 2 & Spherical & Linear limb darkening Eq. (1) \\
Table 3 & Spherical & Quadratic limb darkening Eq. (2) \\
Table 4 & Spherical & Square root limb darkening Eq. (3) \\
Table 5 & Spherical & Four-parameter limb darkening Eq. (4) \\
Table 6 & Spherical & Exponential limb darkening Eq. (5) \\
Table 7 & Spherical & Logarithmic limb darkening Eq. (6) \\
Table 8 & Planar & Linear limb darkening Eq. (1) \\
Table 9 & Planar & Quadratic limb darkening Eq. (2) \\
Table 10 & Planar & Square root limb darkening Eq. (3) \\
Table 11 & Planar & Four-parameter limb darkening Eq. (4) \\
Table 12 & Planar & Exponential limb darkening Eq. (5) \\
Table 13 & Planar & Logarithmic limb darkening Eq. (6) \\
Table 14 & Spherical & Gravity darkening \\
Table 15 & Planar & Gravity darkening \\
Table 16 & Spherical & Angular diameter corrections \\
Table 17 & Planar & Angular diameter corrections \\
\hline
\end{tabular}

Notes. Tables listed here can be retrieved electronically from the CDS.

intensity profile and limb-darkened angular diameter in modeling interferometric observations. Therefore, differences between the intensity profiles of plane-parallel and spherically symmetric model stellar atmospheres lead directly to differences between predicted angular diameter corrections. We find that spherically symmetric model corrections are about 1 to $2 \%$ smaller than planar model corrections for the main sequence stars analyzed here.

Similarly, we computed limb-darkening coefficients for six different limb-darkening laws. As in Paper I, we find that the linear law is least consistent with predicted intensity profiles and that the four-parameter law is best. We also find that the commonly used quadratic limb-darkening law does not fit spherically symmetric model atmosphere intensity profiles as precisely as the similar square-root or four-parameter limb-darkening laws. This suggests that as planetary-transit observations become increasingly precise, the four-parameter law combined with the more physically representative spherically symmetric model stellar atmospheres will be more appropriate for fitting observations or, better still, using intensity profiles directly.

The angular-diameter corrections, limb-darkening and gravity-darkening coefficients are publicly available as online tables. Each table has the format $T_{\text {eff }}(\mathrm{K}), \log g$ and $M\left(M_{\odot}\right)$ and then the appropriate variables for each waveband, such as linear limb-darkening coefficients. Tables for plane-parallel model fits do not include mass. Tables of gravity-darkening coefficients also contain values of the intensity derivatives with respect to gravity and effective temperature. For plane-parallel models, values of mass, radius and luminosity are presented as zero in the tables. We list the properties of these tables in Table 1 that are available from CDS. Tabulated grids of the model atmosphere intensity profiles used in this work are also available.

Acknowledgements. This work has been supported by a research grant from the Natural Sciences and Engineering Research Council of Canada, the Alexander von Humboldt Foundation and NSF grant (AST-0807664).

\section{References}

Al-Naimiy, H. M. 1978, Ap\&SS, 53, 181

An, J. H., Albrow, M. D., Beaulieu, J.-P., et al. 2002, ApJ, 572, 521

Auvergne, M., Bodin, P., Boisnard, L., et al. 2009, A\&A, 506, 411

Barros, S. C. C., Pollacco, D. L., Gibson, N. P., et al. 2012, MNRAS, 419, 1248

Bass, G., Orosz, J. A., Welsh, W. F., et al. 2012, ApJ, 761, 157

Bessell, M. S. 2005, ARA\&A, 43, 293

Bloemen, S., Marsh, T. R., Østensen, R. H., et al. 2011, MNRAS, 410, 1787

Boyajian, T. S., von Braun, K., van Belle, G., et al. 2012, ApJ, 757, 112

Claret, A. 2000, A\&A, 363, 1081

Claret, A., \& Bloemen, S. 2011, A\&A, 529, A75

Claret, A., \& Hauschildt, P. H. 2003, A\&A, 412, 241

Claret, A., Hauschildt, P. H., \& Witte, S. 2012, A\&A, 546, A14

Claret, A., Hauschildt, P. H., \& Witte, S. 2013, A\&A, 552, A16

Croll, B., Albert, L., Jayawardhana, R., et al. 2011, ApJ, 736, 78

Davis, J., Tango, W. J., \& Booth, A. J. 2000, MNRAS, 318, 387

Diaz-Cordoves, J., Claret, A., \& Gimenez, A. 1995, A\&AS, 110, 329

Feautrier, P. 1964, Comptes Rendus Académie des Sciences (série non spécifiée), 258, 3189

Fields, D. L., Albrow, M. D., An, J., et al. 2003, ApJ, 596, 1305

Hauschildt, P. H., Allard, F., \& Baron, E. 1999, ApJ, 512, 377

Howarth, I. D. 2011a, MNRAS, 413, 1515

Howarth, I. D. 2011b, MNRAS, 418, 1165

Johnson, H. L., \& Morgan, W. W. 1953, ApJ, 117, 313

Koch, D. G., Borucki, W., Dunham, E., et al. 2004, in SPIE Conf. Ser. 5487, ed. J. C. Mather, 1491

Kurucz, R. L. 1979, ApJS, 40, 1

Lester, J. B., \& Neilson, H. R. 2008, A\&A, 491, 633

Mandel, K., \& Agol, E. 2002, ApJ, 580, L171

Mihalas, D. 1978, Stellar atmospheres, 2nd edn. (San Francisco: W. H. Freeman and Co.)

Neilson, H. R., \& Lester, J. B. 2011, A\&A, 530, A65

Neilson, H. R., \& Lester, J. B. 2012, A\&A, 544, A117

Neilson, H. R., \& Lester, J. B. 2013, A\&A, 554, A98

Rybicki, G. B. 1971, J. Quant. Spec. Radiat. Transf., 11, 589

Sing, D. K. 2010, A\&A, 510, A21

van Hamme, W. 1993, AJ, 106, 2096

von Zeipel, H. 1924, MNRAS, 84, 665

Wade, R. A., \& Rucinski, S. M. 1985, A\&AS, 60, 471 